\title{
EXTENSIONS OF A THEOREM OF WINTNER ON SYSTEMS WITH ASYMPTOTICALLY CONSTANT SOLUTIONS
}

BY

WILLIAM F. TRENCH

\begin{abstract}
A theorem of Wintner concerning sufficient conditions for a system $y^{\prime}=A(t) y$ to have linear asymptotic equilibrium is extended to a system $x^{\prime}=$ $A(t) x+f(t, x)$. The integrability conditions imposed on $f$ permit conditional convergence of some of the improper integrals that occur. The results improve on Wintner's even if $f=0$.
\end{abstract}

An $n \times n$ system

$$
y^{\prime}=A(t) y, \quad t>0,
$$

is said to have linear asymptotic equilibrium if for each constant vector $c$ there is a solution of (1) such that $\lim _{t \rightarrow \infty} y(t)=c$. It is well known that (1) has this property if $A$ is continuous and

$$
\int^{\infty}\|A(t)\| d t<\infty
$$

Wintner [5] attributed this result to Bôcher and improved on it as follows.

ThEOREM 1 (WINTNER). Let $A$ be continuous on $[a, \infty)$ and suppose the integrals

$$
A_{j}(t)=\int_{t}^{\infty} A_{j-1}(s) A(s) d s, \quad 1 \leqslant j \leqslant k\left(A_{0}=I\right),
$$

converge, and

$$
\int^{\infty}\left\|A_{k}(t) A(t)\right\| d t<\infty
$$

Then (1) has linear asymptotic equilibrium.

Notice that (3) is vacuous and (4) reduces to (2) when $k=0$.

Here we apply Wintner's idea to the system

$$
x^{\prime}=A(t) x+f(t, x) .
$$

We give sufficient conditions for (5) to have a solution $x$ such that

$$
\lim _{t \rightarrow \infty} x(t)=c
$$

Received by the editors February 29, 1984.

1980 Mathematics Subject Classification. Primary 34C11, 34D10. 
for a given constant vector $c$. The assumptions on $f$ in our main theorem apply only "near" the set $\{(t, c) \mid t \geqslant a\}$. Our integral smallness conditions permit conditional convergence of some of the improper integrals that occur. This continues a theme developed previously in [3 and 4]. (See also Hallam [1, 2].) The idea of dealing with the iterated integrals (3) is due to Wintner [5].

Throughout the paper $k$ is a nonnegative integer. It is to be understood that conditions stated for $1 \leqslant j \leqslant k$ are vacuous if $k=0$, and that $\sum_{j=1}^{0}=0$. Our results apply with $x, A$, and $f$ real- or complex-valued. Improper integrals occurring in hypotheses are tacitly assumed to converge, and the convergence may be conditional except when the integrand is obviously nonnegative. We use " $O$ " and " $O$ " in the standard way to indicate orders of magnitude as $t \rightarrow \infty$.

To indicate the direction of proof of our main theorem, it is convenient to state part of its hypotheses here.

Assumption A. Let $w$ be continuous and nonincreasing on $[a, \infty), 0<w(t) \leqslant 1$, and suppose that either $\lim _{t \rightarrow \infty} w(t)=0$ or $w=1$. Suppose $A$ is continuous on $[a, \infty)$ and $A_{j}$ in (3) exists for $1 \leqslant j \leqslant k$. Let $c$ be a given constant vector, and suppose there is a constant $M>0$ such that $f$ is continuous and

$$
\|f(t, x)-f(t, c)\| \leqslant R(t,\|x-c\|)
$$

on

$$
S=\{(t, x) \mid t \geqslant a,\|x-c\| \leqslant M w(t)\},
$$

where $R(t, \lambda)$ is continuous on $\{(t, \lambda) \mid t \geqslant a, 0 \leqslant \lambda \leqslant M w(t)\}$ and nondecreasing in $\lambda$ for each $t$.

We define $\Gamma_{k}=\sum_{j=0}^{k} A_{j}$, and observe from (3) that

$$
\Gamma_{k}^{\prime}=-\Gamma_{k-1} A, \quad k \geqslant 0\left(\Gamma_{-1}=0\right) .
$$

Moreover, since $\lim _{t \rightarrow \infty} \Gamma_{k}(t)=I, \Gamma_{k}$ is invertible for large $t$, and $\lim _{t \rightarrow \infty} \Gamma_{k}^{-1}(t)=$ I.

Now let $t_{0} \geqslant a$ be such that $\Gamma_{k}^{-1}$ exists on $\left[t_{0}, \infty\right)$. For convenience below, we define

$$
\mu_{k}(t)=\sup _{s \geqslant t}\left\{\left\|\Gamma_{k}^{-1}(s)\right\|\right\}=1+o(1)
$$

and

$$
\nu_{k}(t)=\mu_{k}(t) \sup _{s \geqslant t}\left\{\left\|\Gamma_{k}(s)\right\|\right\}=1+o(1) .
$$

Let $C\left[t_{0}, \infty\right)$ be the space of continuous $n$-vector functions (with real or complex components) on $\left[t_{0}, \infty\right)$, with the topology of uniform convergence on finite intervals. Let $V\left[t_{0}, \infty\right)$ be the closed convex subset of $C\left[t_{0}, \infty\right)$ defined by

$$
V\left[t_{0}, \infty\right)=\left\{x \in C\left[t_{0}, \infty\right) \mid\|x(t)-c\| \leqslant M w(t), t \geqslant t_{0}\right\} .
$$


We obtain our results by applying the Schauder-Tychonov theorem to an appropriate transformation $T$ of $V\left[t_{0}, \infty\right)$ (for sufficiently large $t_{0}$ ) into itself. To motivate the choice of $T$, we observe that if

$$
x(t)=c-\int_{t}^{\infty}[A(s) x(s)+f(s, x(s))] d s,
$$

where the integral is assumed to converge, then $x$ satisfies (5) and (6). Repeated integration by parts, assuming at each step that $x$ satisfies (5), yields the equation

$$
\Gamma_{k}(t) x(t)=c-\int_{t}^{\infty} A_{k}(s) A(s) x(s) d s-\int_{t}^{\infty} \Gamma_{k}(s) f(s, x(s)) d s .
$$

Although these manipulations are completely formal, (13) suggests the transformation $T$ defined by

$$
(T x)(t)=\Gamma_{k}^{-1}(t)\left[c-\int_{t}^{\infty}\left[A_{k}(s) A(s) x(s)+\Gamma_{k}(s) f(s, x(s))\right] d s\right] .
$$

Assumption A implies that the function $F(t)=f(t, x(t))$ is continuous on $\left[t_{0}, \infty\right)$ if $x \in V\left[t_{0}, \infty\right)$. Hence, if the integrals in (14) converge, differentiation yields

$$
(T x)^{\prime}(t)=\Gamma_{k}^{-1}(t)\left[\Gamma_{k-1}(t) A(t)(T x)(t)+A_{k}(t) A(t) x(t)\right]+f(t, x(t)),
$$

where we have used (9) and the fact that $\left(\Gamma_{k}^{-1}\right)^{\prime}=-\Gamma_{k}^{-1} \Gamma_{k}^{\prime} \Gamma_{k}^{-1}$. Therefore, if $T$ has a fixed point (function) $x_{0}$ in $V\left[t_{0}, \infty\right)$, we see on setting $T x=x=x_{0}$ in (15) that

$$
\begin{aligned}
x_{0}^{\prime}(t) & =\Gamma_{k}^{-1}(t)\left[\Gamma_{k-1}(t)+A_{k}(t)\right] A(t) x_{0}(t)+f(t, x(t)) \\
& =A(t) x_{0}(t)+f(t, x(t))
\end{aligned}
$$

(since $\Gamma_{k-1}+A_{k}=\Gamma_{k}$ ); i.e., $x_{0}$ satisfies (5). Moroever, setting $T x=x=x_{0}$ in (14) shows that $x_{0}$ also satisfies (6).

The following theorem allows the integrals occurring in $T c$ (the function obtained by setting $x=c$ in (14)) to converge conditionally, and exploits the rapidity with which $T c-c$ approaches zero for large $t$ to restrict the set $V\left[t_{0}, \infty\right)$ on which $T$ must satisfy the hypotheses of the Schauder-Tychonov theorem. (See Remark 1, below.)

TheOREM 2. Suppose Assumption A holds. Let

$$
h(t)=\left(\Gamma_{k}^{-1}(t)-I\right) c-\Gamma_{k}^{-1}(t) \int_{t}^{\infty}\left[A_{k}(s) A(s) c+\Gamma_{k}(s) f(s, c)\right] d s,
$$

and suppose that

$$
\varlimsup_{t \rightarrow \infty} \frac{\|h(t)\|}{w(t)}=\alpha .
$$

Suppose also that

$$
\varlimsup_{t \rightarrow \infty} \int_{t}^{\infty}\left[\frac{R(s, M w(s))}{M}+\left\|A_{k}(s) A(s)\right\| w(s)\right] d s=\theta<1
$$

and

$$
\alpha<M(1-\theta) .
$$


Then, if $t_{0}$ is sufficiently large, there is a solution $x_{0}$ of $(5)$ on $\left[t_{0}, \infty\right)$ such that

$$
\left\|x_{0}(t)-c\right\| \leqslant M w(t), \quad t \geqslant t_{0},
$$

and

$$
\varlimsup_{t \rightarrow \infty}(w(t))^{-1}\left\|x_{0}(t)-c\right\| \leqslant \alpha+M \theta ;
$$

or, more precisely,

$$
\varlimsup_{t \rightarrow \infty}(w(t))^{-1}\left\|x_{0}(t)-c-h(t)\right\| \leqslant M \theta .
$$

Proof. We can rewrite (14) as

$$
\begin{aligned}
(T x)(t)=c+h(t)-\Gamma_{k}^{-1}(t) \int_{t}^{\infty}[ & A_{k}(s) A(s)(x(s)-c) \\
& \left.+\Gamma_{k}(s)(f(s, x(s))-f(s, c))\right] d s,
\end{aligned}
$$

where the integral converges if $x \in V\left[t_{0}, \infty\right)$, because of (7), (12), and (18); moreover

$$
\begin{aligned}
\|(T x)(t)-c\| \leqslant & \|h(t)\|+M \mu_{k}(t) \int_{t}^{\infty}\left\|A_{k}(s) A(s)\right\| w(s) d s \\
& +\nu_{k}(t) \int_{t}^{\infty} R(s, M w(s)) d s .
\end{aligned}
$$

From (10), (11), (18), and (19), we can assume henceforth that $t_{0}$ is so large that the right side of (24) is $\leqslant M w(t)$ if $t \geqslant t_{0}$. Then

$$
T\left(V\left[t_{0}, \infty\right)\right) \subset V\left[t_{0}, \infty\right) .
$$

Now we show that $T$ is continuous on $V\left[t_{0}, \infty\right)$. Let $\left\{x_{j}\right\}$ be a sequence in $V\left[t_{0}, \infty\right)$ which converges to a limit $x$ in $V\left[t_{0}, \infty\right)$. From (10), (11), and (23).

$$
\begin{aligned}
\left\|\left(T x_{j}\right)(t)-(T x)(t)\right\| \leqslant & \mu_{k}\left(t_{0}\right) \int_{t_{0}}^{\infty}\left\|A_{k}(s) A(s)\right\|\left\|x_{j}(s)-x(s)\right\| d s \\
& +\nu_{k}\left(t_{0}\right) \int_{t_{0}}^{\infty}\left\|f\left(s, x_{j}(s)\right)-f(s, x(s))\right\| d s, \quad t \geqslant t_{0} .
\end{aligned}
$$

The integrands here converge to zero on $\left[t_{0}, \infty\right)$, and they are dominated by $2 M\left\|A_{k}(s) A(s)\right\| w(s)$ and $2 R(s, M w(s))$, respectively. (See (7) and (12).) Therefore, (18) and Lebesgue's dominated convergence theorem imply that the integrals approach zero as $j \rightarrow \infty$. Hence, $\left\{T x_{j}\right\}$ converges to $T x$ uniformly on $\left[t_{0}, \infty\right)$, and therefore $T$ is continuous on $V\left[t_{0}, \infty\right)$.

From (12) and (25), $T\left(V\left[t_{0}, \infty\right)\right)$ is equibounded on finite intervals. This, (15), and Assumption A imply that $T\left(V\left[t_{0}, \infty\right)\right.$ ) is also equicontinuous on finite intervals. Now we have verified the hypotheses of the Schauder-Tychonov theorem, which implies that $T x_{0}=x_{0}$ for some $x_{0}$ in $V\left[t_{0}, \infty\right)$. Setting $T x=x_{0}$ in (24) and invoking (10), (11), (17), and (18) yields (20). Similarly, setting $x=T x=x_{0}$ in (23) yields (22). This completes the proof. 
COROLlaRY 1. In addition to the assumptions of Theorem 2, suppose that

$$
R\left(t, \lambda_{1}\right) / R\left(t, \lambda_{2}\right) \leqslant \lambda_{1} / \lambda_{2}, \quad 0 \leqslant \lambda_{1}<\lambda_{2} .
$$

Then (21) can be replaced by

$$
\varlimsup_{t \rightarrow \infty}(w(t))^{-1}\left\|x_{0}(t)-c\right\| \leqslant \alpha /(1-\theta) .
$$

Proof. Let

$$
\phi(t)=\sup _{s \geqslant t}\left\{(w(s))^{-1}\left\|x_{0}(s)-c\right\|\right\}
$$

and

$$
\delta=\lim _{t \rightarrow \infty} \phi(t)=\varlimsup_{t \rightarrow \infty}(w(t))^{-1}\left\|x_{0}(t)-c\right\| .
$$

Setting $x=T x=x_{0}$ in (23) and using routine estimates yields

$$
\begin{aligned}
\left\|x_{0}(t)-c\right\| \leqslant & \|h(t)\|+\nu_{k}(t) \int_{t}^{\infty}\left\|A_{k}(s) A(s)\right\|\left\|x_{0}(s)-c\right\| d s \\
& +\mu_{k}(t) \int_{t}^{\infty} R\left(s,\left\|x_{0}(s)-c\right\|\right) d s .
\end{aligned}
$$

Applying (26) in the second integral and then dividing (29) by $w(t)$ yields the inequality

$$
(w(t))^{-1}\left\|x_{0}(t)-c\right\| \leqslant(w(t))^{-1}\|h(t)\|+P(t) \phi(t),
$$

where

$$
P(t)=(w(t))^{-1}\left[\nu_{k}(t) \int_{t}^{\infty}\left\|A_{k}(s) A(s)\right\| w(s) d s+\frac{\mu_{k}(t)}{M} \int_{t}^{\infty} R(s, M w(s)) d s\right],
$$

so that

$$
\lim _{t \rightarrow \infty} P(t)=\theta \text {, }
$$

from (10), (11), and (18). Letting $t \rightarrow \infty$ in (30) and invoking (17), (28), and (31) shows that $\delta \leqslant \alpha+\theta \delta$, which proves (27).

It is worthwhile to state Theorem 2 separately for the case where $w=1$, so that $\alpha$ and $\theta$ are necessarily zero and (19) is automatic.

THEOREM 3. Suppose Assumption A holds with $w=1$. Suppose also that the integral in (16) converges and that

$$
\int^{\infty} R(t, M) d t<\infty, \quad \int^{\infty}\left\|A_{k}(t) A(t)\right\| d t<\infty
$$

Then, if $t_{0}$ is sufficiently large, there is a solution $x_{0}$ of $(5)$ on $\left[t_{0}, \infty\right)$ such that $\left\|x_{0}(t)-c\right\| \leqslant M$ for $t \geqslant t_{0}$ and $\lim _{t \rightarrow \infty} x_{0}(t)=c$.

REMARK 1. The continuity assumption on $f$ is the most stringent when $w=1$, since the set $S$ in (8) is maximized in that case. More importantly, if $R$ satisfies (26), then (32) implies (18) with $\theta=0$ for every admissible $w \neq 1$, while the converse is obviously false. Nevertheless, the conclusions of Theorem 2 are weakest when 
$w=1$. (See Theorem 3.) The reason for this is that in Theorem 3 it is assumed only that the integral in (16) converges, while Theorem 2 exploits the rapidity of its convergence.

The hypotheses of Theorem 2 may hold for some constant vectors $c$ and fail to hold for others. In the following theorem $c$ may be chosen arbitrarily.

THEOREM 4. Suppose $f$ is continuous for $t \geqslant a$ and all $x$, and

$$
\left\|f\left(t, x_{1}\right)-f\left(t, x_{2}\right)\right\| \leqslant R\left(t,\left\|x_{1}-x_{2}\right\|\right),
$$

where $R(t, \lambda)$ is continuous on $[a, \infty) \times[0, \infty)$ and nondecreasing in $\lambda$. For some integer $k \geqslant 0$, suppose the integrals $A_{1}, \ldots, A_{k+1}$ converge, and

$$
\left\|A_{j}(t)\right\|=O(w(t)), \quad 1 \leqslant j \leqslant k+1 .
$$

Suppose also that

$$
\left\|\int_{t}^{\infty} \Gamma_{k}(s) f(s, c) d s\right\|=O(w(t))
$$

for every constant vector $c$, and that (18) holds for all $M>0$. Then, if $c$ is a given constant vector, there is a solution $x_{0}$ of (5) which is defined for $t$ sufficiently large and satisfies

$$
\left\|x_{0}(t)-c\right\|=O(w(t)) .
$$

Moreover, if $\theta=0$ in (18) and (33) and (34) hold with " $O$ " replaced by " $O$ " (which is necessarily true if $w=1)$, then (35) holds with " $O$ " replaced by " $O$."

Proof. The hypotheses imply (17) for some $\alpha$ (which may depend upon $c$, but is zero if (33) and (34) hold with " $o$ "). Simply choose $M$ to satisfy (19) and invoke Theorem 2.

Theorem 4 has the following corollary for the linear system

$$
x^{\prime}=[A(t)+B(t)] x+g(t) .
$$

Corollary 2. Let $A, B$, and $g$ be continuous on $[a, \infty)$. Suppose (33) holds.

$$
\left\|\int_{t}^{\infty} \Gamma_{k}(s) g(s) d s\right\|=O(w(t)),\left\|\int_{t}^{\infty} \Gamma_{k}(s) B(s) d s\right\|=O(w(t)) .
$$

and

$$
\varlimsup_{t \rightarrow \infty}(w(t))^{-1} \int_{t}^{\infty}\left[\left\|A_{k}(s) A(s)\right\|+\|B(s)\|\right] w(s) d s=\theta<1 .
$$

Then, for any constant $c$, (36) has a solution which satisfies (35); moreover, if $\theta=0$ and (33) and (37) hold with " $O$ " replaced by " $o$," then so does (35).

The following special case of Corollary 2 extends Theorem 1 .

Corollary 3. Suppose (33) holds and

$$
\varlimsup_{t \rightarrow \infty}(w(t))^{-1} \int_{t}^{\infty}\left\|A_{k}(s) A(s)\right\| w(s) d s<1
$$

for some $w$ as in Assumption A. Then (1) has linear asymptotic equilibrium. 


\section{BIBLIOGRAPHY}

1. T. G. Hallam, Asymptotic integration of second order differential equations with integrable coefficients, SIAM J. Appl. Math. 19 (1970), 430-439.

2. Asymptotic integration of a nonhomogeneous differential equation with integrable coefficients, Czechoslovak Math. J. 21 (1971), 661-671.

3. W. F. Trench, Systems of differential equations subject to mild integral conditions, Proc. Amer. Math. Soc. 87 (1983), 263-270.

4. Asymptotics of differential systems with deviating arguments, Proc. Amer. Math. Soc. 92 (1984), 219-224.

5. A. Wintner, On a theorem of Bôcher in the theory of ordinary linear differential equations, Amer. J. Math. 76 (1954), 183-190.

Department of Mathematics and Computer Science, Drexel. University, Philadelphia, PennSYLVANIA 19104 